\title{
Management der ersten COVID-19-Welle in 45 Kinder- und Jugendarztpraxen im Saarland
}

\section{Management of the First COVID-19 Wave in 45 Pediatric Practices in Saarland}

\author{
Autoren \\ Karsten Theiß ${ }^{1}$, Arne Simon ${ }^{2}$, Norbert Graf², Tilman Rohrer ${ }^{3}$
}

Institute

1 Kinder- und Jugendmedizin, Kinder- und Jugendarztpraxis Theiß, St. Ingbert, Deutschland

2 Klinik für Pädiatrische Onkologie und Hamätologie, Universitätsklinikum des Saarlandes und Medizinische Fakultät der Universität des Saarlandes, Homburg, Deutschland

3 Klinik für Allgemeine Pädiatrie und Neonatologie, Universitätsklinikum des Saarlandes und Medizinische Fakultät der Universität des Saarlandes, Homburg, Deutschland

\section{Schlüsselwörter}

COVID-19, Pädiatrie, Infektionsschutz, Praxismanagement, Zusammenarbeit, Gesundheitswesen

Key words

COVID-19, Pediatrics, Public health, Practice management, Child day care

online publiziert $\quad 15.03 .2021$

Bibliografie

Gesundheitswesen 2021; 83: 258-264

DOI 10.1055/a-1384-0568

ISSN 0941-3790

(C) 2021. Thieme. All rights reserved.

Georg Thieme Verlag KG, Rüdigerstraße 14,

70469 Stuttgart, Germany

\section{Korrespondenzadresse}

Karsten Theiß

Kinder- und Jugendmedizin

Kinder- und Jugendarztpraxis Theiß

Oststraße 68

66386 St. Ingbert

Deutschland

karsten.theiss@kinder-und-jugendarzt-theiss.de

$\Theta$

Zusätzliches Material zu diesem Beitrag finden Sie unter https://doi.org/10.1055/a-1384-0568.

\section{ZUSAMMENFASSUNG}

Hintergrund Das Saarland ist in der ersten Welle der COVID19-Pandemie eines der am stärksten betroffenen Bundesländer. Niedergelassene Kinder- und Jugendärzte sind für pädiatrische Patienten und ihre Familien erste Ansprechpartner.

Fragestellung Darstellung der Herausforderungen und Maßnahmen zur Aufrechterhaltung der Patientenversorgung sowie der Zusammenarbeit während der COVID-19-Pandemie. Methoden Internet-basierte Befragung der niedergelassenen Kinder- und Jugendärzte sowie papierbasierte Befragung von nicht-ärztlichem Assistenzpersonal der Kinder- und Jugendarztpraxen im Saarland.

Ergebnisse Inhaber von $85 \%$ sowie Assistenzpersonal aus $81 \%$ der Praxen nahmen teil. Für $71 \%$ der Praxisinhaber bzw. 48 \% des Assistenzpersonals bestand ein erhöhtes persönliches Ausfallrisiko als Risikogruppenangehörige oder aufgrund von Betreuungsverpflichtungen. Es kam aber nur zu wenigen tatsächlichen Ausfällen. 85\% halten die Hygiene- und Arbeitsschutzempfehlungen für sinnvoll, aber nur $32 \%$ stand bei Pandemiebeginn die notwendige Schutzausrüstung zur Verfügung. $89 \%$ der Praxen haben Ihr Praxis- und Patientenmanagement in der Pandemie umgestellt. Es wird ein deutlicher Verbesserungsbedarf in der Pandemievorbereitung (77\%) und -bewältigung (61\%), aber auch in der Zusammenarbeit mit dem öffentlichen Gesundheitswesen (59\%) sowie mit Kindertagesstätten und Schulen (77\%) gesehen. Schlussfolgerung Die erste Welle der Pandemie hat die Praxen vor erhebliche Herausforderungen gestellt, die durch betrieblich-funktionelle Umstrukturierung und -organisation bewältigt wurden. Jedoch wird eine bessere Pandemievorbereitung und Unterstützung bei der Bewältigung einschl. verbesserter Zusammenarbeit mit den Gesundheitsämtern und Kinderbetreuungseinrichtungen gefordert.

\section{ABSTRACT}

Background Saarland was one of the most severely affected federal states during the first wave of COVID-19 pandemic. Pediatrician practices are the first point of contact for pediatric patients and their families.

Objectives The aim of the study was to describe the challenges and actions taken for maintenance of patient care as well as the interdisciplinary cooperation during the COVID-19 pandemic. 
Materials and methods Interned-based survey of pediatrician practice owners as well as paper-based survey of nonphysician healthcare professionals (assistant personnel) of practices in Saarland.

Results In this survey, the percentage of participation was $85 \%$ of owners of the 53 practices as well as $81 \%$ of the assistant personnel. An increased risk of being absent from work existed for $71 \%$ of practice owners and $48 \%$ of assistant personnel, either because they belonged to a group at high risk of being infected or due to other care obligations. However, there were very few cases of care givers being actually absent. Although at the beginning of the pandemic, necessary safety equipment was available only for $32 \%, 85 \%$ considered the hygienic and occupational health and safety measure reasonable. During the pandemic, $89 \%$ of practices changed their practice and patient management. There was a clear need for improvement regarding preparation (77\%) and mastering (61\%) the pandemic also regarding collaboration with the public health system (59\%), as well as day care centers and schools (77\%).

Conclusion During the first wave of the pandemic, practices faced significant challenges that were mastered by operational-functional restructuring and reorganization. However, better preparation to deal with the pandemic and support for mastering it including improved collaboration with local health authorities and children's community facilities is required.

\section{Einleitung}

Eine Pandemie stellt Praxen vor erhebliche Herausforderungen, dieses wurde bereits am Beispiel der H1N1-Pandemie 2009/2010 untersucht [1-3].

Am 30.01.2020 informierten sich die saarländischen Kinder- und Jugendärzte in einer Fortbildungsveranstaltung mit dem Institut für Virologie am Universitätsklinikum des Saarlandes (UKS) erstmals über das neue Coronavirus. Am 03.03.2020 kam es im Saarland zum ersten Erkrankungsfall. Vom 11.03. - 25.09.2020 lag die kumulierte Inzidenz über dem Bundesdurchschnitt. Die 7-Tage-Inzidenz erreichte Ihren Höhepunkt in der ersten Welle am 05.04.2020 mit 82,4 Infektionen.

In den Kalenderwochen (KW) 12-18 wurden durch die Landesregierung Ausgangs- und Kontaktbeschränkungen verfügt, die auch die Schließung von Schulen und Kindertageseinrichtungen (Kitas) beinhalteten.

Dieser Beitrag stellt anhand eines Internet-basierten Surveys den Umgang der niedergelassenen Kinder- und Jugendärzte im Saarland mit der ersten Welle der COVID-19-Pandemie im Kontext von Arbeitsschutz \& Hygiene, Sprechstundenorganisation \& Patientensteuerung, Auswirkungen des Lockdowns (KW 12-18) im Sinne von Kollateralschäden, Informationsquellen, Zusammenarbeit \& Netzwerke sowie wirtschaftliche Auswirkungen auf die Praxis dar.

\section{Methodik}

Im Saarland sind 52 Praxen sowie ein Medizinisches Versorgungszentrum zur vertragsärztlichen Versorgung in der Kinder- und Ju-

- Tab. 1 Verteilung der Praxen. Verteilung der Praxen auf die Landkreise (=Zulassungsbezirke) nach Zulassung sowie Teilnahme am jeweiligen Survey.

\begin{tabular}{|l|l|l|}
\hline & $\begin{array}{l}\text { Praxisinhaber } \\
\text { Anteil } \\
\text { Zulassungen/ } \\
\text { Anteil Teilnehmer }\end{array}$ & $\begin{array}{l}\text { med. Assistenz- } \\
\text { personal Anteil } \\
\text { Zulassungen/ } \\
\text { Anteil Teilnehmer }\end{array}$ \\
\hline Regionalverband Saarbrücken & $41,5 \% / 37,8 \%$ & $42,3 \% / 40,5 \%$ \\
\hline Landkreis Saarlouis & $22,6 \% / 20,0 \%$ & $23,1 \% / 23,8 \%$ \\
\hline Landkreis Saarpfalz & $11,3 \% / 13,3 \%$ & $11,5 \% / 9,5 \%$ \\
\hline Landkreis Neunkirchen & $9,4 \% / 11,1 \%$ & $7,7 \% / 9,5 \%$ \\
\hline Landkreis Merzig-Wadern & $9,4 \% / 11,1 \%$ & $9,6 \% / 9,5 \%$ \\
\hline Landkreis St. Wendel & $5,7 \% / 6,7 \%$ & $5,8 \% / 7,1 \%$ \\
\hline
\end{tabular}

gendmedizin zugelassen (Stand: 30.06.2020). Diese 53 Einrichtungen wurden im Zeitraum 17.08.-15.09.2020 zu 30 Items mittels einer Online-Erhebung (Plattform SurveyMonkey ${ }^{\mathrm{TM}}$ ) zu Ihren $\mathrm{Er}$ fahrungen in der ersten Welle der COVID-19-Pandemie befragt (siehe online Supplement).

Der verwendete Fragenkatalog basiert zum Teil auf einem bereits in der H1N1 eingesetzten Fragebogen [2], sodass sich die Möglichkeit einer vergleichenden Auswertung für diese Fragen ergibt.

Parallel wurde im gleichen Befragungszeitraum das nichtärztliche Assistenzpersonal, v. a. die Arzthelferinnen ${ }^{1}$ bzw. Medizinischen Fachangestellten, in 52 Praxen (eine Praxis hat Ihre Tätigkeit zwischenzeitlich eingestellt) mittels eines Papierfragebogens zu ihren Erfahrungen während der Pandemie befragt (siehe online Supplement).

Die Ergebnisse wurden in Microsoft Exce ${ }^{\mathrm{TM}}$ dokumentiert und mittels deskriptiver Statistik ausgewertet.

\section{Ergebnisse}

An der Befragung nahmen 45 der 53 Praxen teil (85\%). Beim Assistenzpersonal wurden 181 Fragebögen aus 42 von 52 Praxen zurückgesendet (Teilnehmerquote $81 \%$ ).

Die Verteilung der teilnehmenden Praxen auf die Landkreise bzw. den Regionalverband Saarbrücken ist in beiden Umfragen deckungsgleich mit der Verteilung zur vertragsärztlichen Versorgung ( Tab. 1).

\section{Sicherstellung des Praxisbetriebs}

Angehörige einer Risikogruppe für einen komplizierten Verlauf einer SARS-CoV-2-Infektion (nach RKI-Kriterien) sind 23 (51\%) der Praxisinhaber und 37 (21\%) des Assistenzpersonals.

Betreuungsverpflichtungen (Kinder, Familie, pflegebedürftige Angehörige, usw.) und zusätzlichen Rückgriff auf unterstützende Ressourcen gaben 15 (34\%) Praxisinhaber und 62 (35\%) des Assistenzpersonals an. Im Lockdown wäre sonst der Praxisbetrieb nicht sichergestellt gewesen.

Wenn man beide Items verbindet, ergeben sich für 32 (71\%) der Praxisinhaber sowie für 86 (48\%) des Assistenzpersonals ein poten-

1 Bei allen Bezeichnungen sind immer alle Geschlechter gemeint. 
- Tab. 2 Vergleich COVID-19-Pandemie und H1N1-Pandemie 2009/201.

\begin{tabular}{|c|c|c|}
\hline & $\begin{array}{l}\text { COVID-19 } 1 . \\
\text { Welle }\end{array}$ & $\begin{array}{l}\text { pH1N1 2009/2010 aus Rexroth et. al. [2], nur Pädiater } \\
\text { ergänzt um persönliche Mitteilungen der Autorin }\end{array}$ \\
\hline \multicolumn{3}{|l|}{ Die offiziellen Arbeitsschutz- und \& Hygieneempfehlungen } \\
\hline ... sind sinnvoll & $86 \%$ & $53 \%$ \\
\hline ... sind praktikabel & $57 \%$ & $32 \%$ \\
\hline Änderungen am Praxismanagement vorgenommen & $89 \%$ & $60 \%$ \\
\hline Räumliche Trennung von (Verdachts)Fällen & $82 \%$ & $62 \%$ \\
\hline ... durch separaten Eingang & $33 \%$ & $37 \%$ \\
\hline ... durch separate Anmeldung & $26 \%$ & $19 \%$ \\
\hline ... durch getrennte Wartezimmer & $80 \%$ & $68 \%$ \\
\hline ... durch getrennte Behandlungsräume & $92 \%$ & $86 \%$ \\
\hline Zeitliche Trennung von (Verdachts)Fällen & $80 \%$ & $25 \%$ \\
\hline ... als Angebot „Fiebersprechstunde“ & $66 \%$ & $71 \%$ \\
\hline ... als Angebot „Vorsorgesprechstunde“ & $83 \%$ & $44 \%$ \\
\hline Anmeldepflicht vor Vorstellung & $100 \%$ & $82 \%$ \\
\hline ... davon neu eingeführt & $60 \%$ & $14 \%$ \\
\hline
\end{tabular}

zielles Ausfallrisiko. Vier der 53 Praxen wurden im zweiten Quartal vorübergehend aufgrund der Pandemie geschlossen.

In 19 von 44 Praxen musste Praxispersonal pandemiebedingt vorübergehend freigestellt werden.

\section{Arbeitsschutz \& Hygiene}

Für das erste Quartal 2020 gaben nur 14 von 44 Praxisinhabern an, dass ausreichend persönliche Schutzausrüstung (PSA) zur Verfügung stand.

Neben zentralen Beschaffungen und Verteilungen von Schutzmaterialien haben 38/44 Praxisinhabern Material zusätzlich auf dem freien Markt erworben ( $5 x$ keine Verfügbarkeit). Die Kosten beliefen sich im Median auf 300 Euro (20-3000€).

Im August 2020 gaben 40 von 44 Praxen an, dass ausreichend PSA vorhanden sei. Bei fehlendem Material wurden Schutzkittel an erster Stelle genannt, gefolgt von Atemschutz sowie Händedesinfektionsmittel.

In 24 der 44 teilnehmenden Praxen wurden SARS-CoV-2 Abstriche überwiegend in der Praxis durchgeführt. Weitere 18 Praxen haben hierfür überwiegend an Dritte verwiesen, v. a. an die Testzentren bzw. Testteams der Kassenärztlichen Vereinigung Saarland (KVS). Bei Praxen, die bereits im ersten Quartal ausreichend PSA hatten, haben 11 von 14 (79\%) Abstriche überwiegend in der Praxis durchgeführt, wohingegen es bei der Gruppe ohne ausreichende PSA nur 13 von 30 (43\%) waren.

Die Mehrheit der Praxisinhaber (38 von 44) halten die aktuellen Arbeitsschutz- und Hygieneempfehlungen für sinnvoll, 25/44 auch für praktikabel und lediglich 9/44 für überzogen.

38 von 43 der Praxisinhaber gaben an, bei der SARS-CoV-2 Testung immer oder häufig PSA zu tragen sowie 40/44 bei Kontakt zu vermutlich infektiösen Patienten. Beim allgemeinen Patientenkontakt wurde in 21/43 Praxen immer oder häufig PSA getragen.

\section{Patientensteuerung \& Sprechstundenorganisation}

Im Rahmen der Pandemie galt in allen Praxen eine telefonische Anmeldepflicht (40\% vor und zusätzlich $60 \%$ während der Pandemie).
Bei 39 von 44 wurde das Praxis- und Sprechstundenmanagement angepasst ( $\triangleright$ Tab. 2 ), weitere Details hierzu sind in $>$ Tab. 3 zusammengestellt.

In 27 von 44 Praxen war die vorübergehende Absage oder Aufschiebung (>2 Wochen) medizinischer Leistungen erforderlich (in 22/44 Kindervorsorgeuntersuchungen, in 19/44 Impftermine, in 19/44 sonstige Leistungen wie z. B. Ultraschalldiagnostik, Lungenfunktion, EKG, Labor, usw. sowie in 16/44 routinemäßige Verlaufskontrollen). 18 der 44 der Praxen führten im Lockdown bzw. nach dessen Aufhebung ein aktives Einbestellen (Recall) durch.

\section{Kollateralschäden des Lockdowns}

Nach Auffassung von 30 der 44 Praxisinhaber gab es bei den eigenen Patienten (Familien) Hinweise auf Kollateralschäden durch den Lockdown ( Tab. 4).

\section{Informationsquellen, Zusammenarbeit \& Netzwerke}

Für Informationen über die regionale Situation der COVID-19-Pandemie wurden durch die 44 Praxisinhaber folgende Informationsquellen regelmäßig genutzt: 44 Info-Fax der KVS, 37 Homepage der KVS, 37 Intranet des Berufsverbandes der Kinder- und Jugendärzte (bvkj) - PädInform ${ }^{\mathrm{TM}}$, 32 WhatsApp ${ }^{\mathrm{TM}}$-Gruppe des bvkj Saarland, 30 Infostream/Videofortbildung von Ärztekammer (ÄKS), KVS und UKS, 24 Homepage des saarländischen Gesundheitsministeriums bzw. Themenportal der saarländischen Landesregierung sowie 18 Homepage der ÄKS incl. dort abgelegter FAQ-Listen.

Die Zusammenarbeit mit dem Berufsverband und der KVS war für 27 (bzw. 31) von 44 Praxisinhabern problemlos. Eine problemlose Zusammenarbeit mit den zuständigen Gesundheitsämtern attestierten nur 11/44 der Praxisinhaber, hier war die Variabilität in Abhängigkeit der Praxislage hoch (0-50\% der Teilnehmer).

Drei Viertel der Praxisinhaber $(n=34 / 44)$ sehen in der Pandemievorbereitung Verbesserungsbedarf. Für die Pandemiebewältigung sehen den Verbesserungsbedarf 27 Teilnehmer. Eine verbesserte Zusammenarbeit mit dem ÖGD erwarten 26 (Spannweite ja nach Praxislage von 20-100\% der Teilnehmer). Die Zusammen- 
- Tab. 3 Patientensteuerung und Sprechstundenorganisation.

\begin{tabular}{|c|c|}
\hline & Anteil der Praxen \\
\hline \multicolumn{2}{|l|}{ Einführung bzw. starke Erweiterung von } \\
\hline ... Telefonsprechstunde & $18 \%$ \\
\hline ... Videosprechstunde & $23 \%$ \\
\hline \multicolumn{2}{|l|}{ Informationsweg über geänderte Abläufe: } \\
\hline - persönlich während Konsultationen & $100 \%$ \\
\hline - Aushänge in den Praxisräumen & $77 \%$ \\
\hline - Praxishomepage & $26 \%$ \\
\hline - Anrufbeantworteransagen & $18 \%$ \\
\hline - soziale Medien & $16 \%$ \\
\hline Vorübergehende Absage oder Aufschiebung ( $>2$ Wochen) von medizinischen Leistungen & $61 \%(n=27)$ \\
\hline \multicolumn{2}{|l|}{ darunter } \\
\hline ... Kindervorsorgeuntersuchungen & $81 \%$ \\
\hline ... Schutzimpfungen & $70 \%$ \\
\hline ... sonstige Leistungen (z. B. Ultraschalldiagnostik, Lungenfunktion, EKG, Labor, usw.) & $70 \%$ \\
\hline ... routinemäßige Verlaufskontrollen & $59 \%$ \\
\hline Durchführung eines aktiven Einbestellens (Recall) um Leistungen nachzuholen & $41 \%(n=18)$ \\
\hline
\end{tabular}

- Tab. 4 Beobachtete Auswirkungen (Kollateralschäden) des Lockdowns.

\begin{tabular}{|l|l|}
\hline & Anteil zustimmender Praxen \\
\hline Fehlende Förder-/Therapiemöglichkeiten für Kinder mit Einschränkungen & $97 \%$ \\
\hline Absage wichtiger Vorsorge-/Impftermine durch Eltern & $90 \%$ \\
\hline massive innerfamiliäre/häusliche Belastungssituationen & $87 \%$ \\
\hline Belastungsbedingte Verhaltensauffälligkeiten bei Kindern & $87 \%$ \\
\hline Absage wichtiger Verlaufskontrollen durch Eltern & $83 \%$ \\
\hline zu späte Konsultationen trotz akuter schwerwiegender Erkrankungen & $17 \%$ \\
\hline Kindesmissbrauch oder -misshandlung & $13 \%$ \\
\hline
\end{tabular}

arbeit mit Kindertagesstätten und Schulen halten 34 für verbesserungswürdig ( $\triangleright$ Abb. 1 und online Supplement).

\section{Wirtschaftliche Auswirkungen auf die Praxis}

Von 44 Praxisinhabern gaben 8 an, dass sie neben dem Schutzschirm gemäß § 87a Abs. 3b S. 3 SGB V weitere finanzielle Hilfen (z. B. Kurzarbeitergeld, Soforthilfen, Kredite, Personalabbau, usw.) für die Sicherung der Praxisexistenz in Anspruch genommen haben.

14 von 44 empfinden die wirtschaftlichen Auswirkungen als persönliche Belastung.

In der Gruppe des nicht-ärztlichen Assistenzpersonals gaben 30 (17\%) an aufgrund der Pandemiesituation finanzielle Einbußen zu haben.

\section{Belastungen des nicht-ärztlichen Assistenzpersonals}

102 (57\%) der Teilnehmer gaben an, Angst um ihre eigene Gesundheit (vor einer schweren SARS-CoV-2 Infektion) oder um die Gesundheit ihrer Familie haben.

Vorbehalten, Anfeindungen oder Stigmatisierungen in Ihrem privaten Umfeld, aufgrund ihrer beruflichen Tätigkeit, waren 33 (18\%) der Teilnehmer an der Befragung des Assistenzpersonals ausgesetzt.

\section{Diskussion}

\section{Versorgungssicherheit \& Infektionsschutz}

Die Versorgungssicherheit der Patienten bzw. die Wahrung des Sicherstellungsauftrages für die ambulante Pädiatrie hängt zum einen von den Personen und zum anderen von den (Verbrauchs-) Materialien für einen sicheren Praxisbetrieb ab. In der aktuellen Befragung war bei $71 \%$ der Praxisinhaber sowie $41 \%$ des Assistenzpersonals ein persönliches Ausfallrisiko (Risikogruppe/Betreuungsverpflichtung) zu ermitteln. Mit Stand 01.07.2020 sind 64,6\% der Fachgruppenangehörigen der Kinder- und Jugendmedizin im Saarland über 50 Jahre alt, 25,6\% sind über 60 Jahre (persönliche Mitteilung KVS).

Die Versorgung mit Schutzausrüstung war in der initialen Phase für die Praxen eine große Herausforderung, nur ca. 1/3 der Praxen stand im ersten Quartal ausreichend Ausrüstung zur Verfügung. Die KVS hat ab 05.03.2020 einen mobilen Abstrichentnahmedienst eingeführt, der ab dem 16.03.2020 in stationäre Testzentren überführt wurde. Dieses Angebot wurde von 41 \% der Praxen genutzt, jedoch muss die Indikationsstellung und die Untersuchung der Kinder weiterhin in der Praxis erfolgen. Ein Bezug von Schutzausrüstung auf dem freien Markt war nicht immer bzw. wenn, dann nur zu deutlich erhöhten Bezugskonditionen möglich. Ab dem 


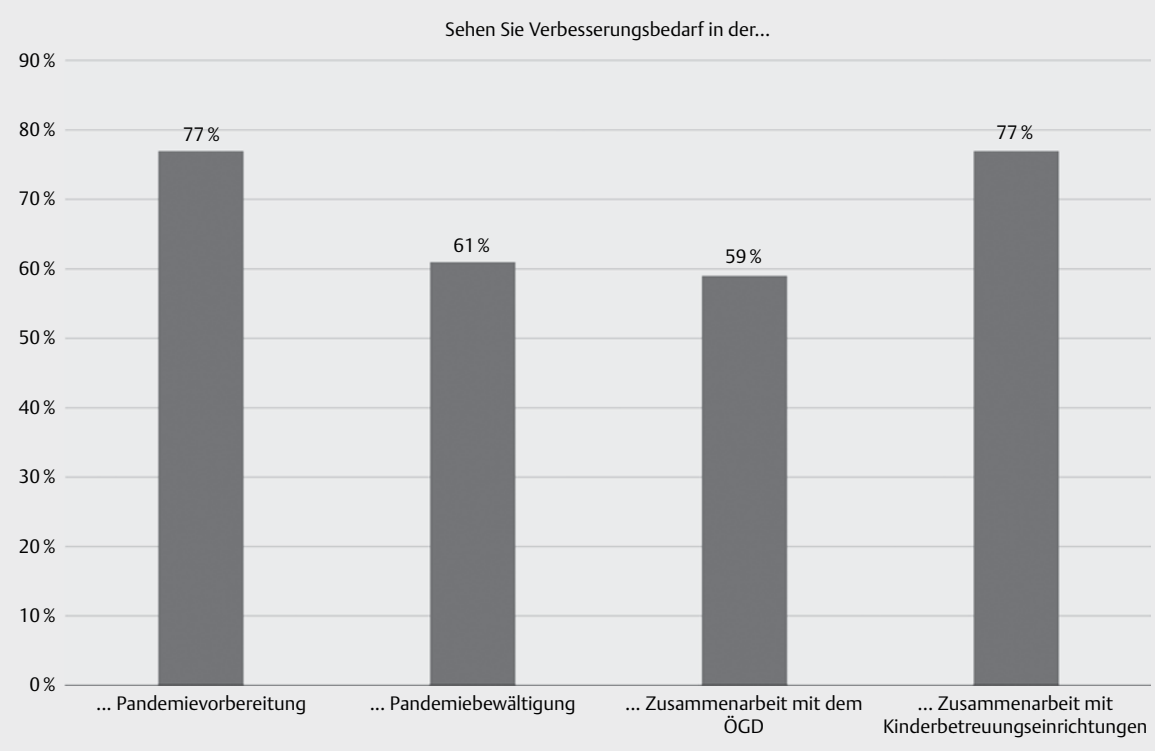

Abb. 1 Angabe von Verbesserungsbedarf durch die Praxisinhaber.

27.03.2020 bestand die Möglichkeit über die KVS Schutzausrüstung zu beziehen. Ab Mitte April besserte sich die Verfügbarkeit von Mund-Nasen-Schutz und FFP-Masken, bei Schutzkitteln bestand nach wie vor ein Lieferengpass.

Im bvkj Saarland sprach sich die Mehrheit gegen die Einrichtung von „Fieberpraxen“ für Kinder und Jugendliche aus. Ein pädiatrischer Fieberpraxisbetrieb wäre nur unter Umverteilung von Versorgungsressourcen, unter Einschränkung der Regelversorgung, möglich.

\section{Praxisorganisation}

Es wurden erhebliche Anstrengungen und Umstrukturierungen der Praxisorganisation vorgenommen, um die Patientenversorgung sicherzustellen. Ein besonderes Augenmerk wurde hierbei auf die Versorgung von COVID-19-(Verdachts-)Fällen gelegt. Alle Praxen haben weiterhin Vorsorgeuntersuchungen und Schutzimpfungen angeboten sowie chronisch kranke Kinder und Jugendliche betreut [4]. Im Vergleich zur H1N1-Pandemie 2009/2010 [2], haben aktuell mehr Praxen Ihr Praxismanagement umgestellt ( Tab. 2).

\section{Kollateralschäden durch COVID-19-Pandemie und Lockdown}

Mögliche Kollateralschäden durch den Lockdown betrafen in 17\% Komplikationen durch zu späte Vorstellungen bei akuten Erkrankungen. In Italien ist eine verspätete Inanspruchnahme von medizinischer Hilfe aus Angst vor COVID-19 gezeigt worden [5]. In anderen Arbeiten werden deutliche Auswirkungen der COVID19-Pandemie wie erhöhte Rate an diabetischer Ketoazidose im Rahmen der Diabetes Mellitus Erstmanifestation beschrieben [6] oder auch verspätete Appendizitis-Diagnosen und -Behandlungen [7].

In einem hohen Prozentsatz wurden innerfamiliäre Belastungssituationen während des Lockdowns berichtet, z. B. finanzielle Sorge, Homeschooling-Situation usw. und auch belastungsbe- dingte Verhaltensauffälligkeiten. Diese Situation ist derzeit Gegenstand intensiver internationaler Forschung [8, 9]. Entsprechend klar sind die Forderungen, den Kindern und Jugendlichen ihre Alltagsstrukturen zu erhalten $[10,11]$. Für chronisch erkrankte Kinder und Kinder mit Beeinträchtigungen sind zusätzlich sehr häufig elementare, medizinisch dringend erforderliche Förder- und Therapieangebote pausiert worden.

Die Situation von Kindesmissbrauch und -misshandlung in der Lockdown-Situation wurde nicht nur im Survey von den niedergelassenen Kinder- und Jugendärzten angegeben, sondern auch an der Kinderklinik des UKS beobachtet [12]. Ob ein ursächlicher Zusammenhang besteht, ist derzeit noch Gegenstand wissenschaftlicher Überprüfung.

\section{Informationsquellen und Netzwerke}

Als Informationsquelle zur nationalen Situation und Empfehlungen ist unstrittig das Internetportal des RKI an erster Stelle.

Alle befragten Kolleginnen und Kollegen nutzten das KVS-InfoFax als primäre Informationsquelle. Daneben wurden in absteigender Reihenfolge als primäre Informationsquellen die Homepage der KVS, das Internetthemenportal der Landesregierung/Saarländisches Gesundheitsministerium sowie die Homepage der ÄKS genutzt. Diese Angebote erfordern jedoch eine aktive Informationssuche. Eine besonders positive Rolle kam ab dem 05.03.2020 dem von Dr. Jürgen Rissland koordinierten regionalen Videocast von ÄKS, KVS und UKS zu.

Eine zusätzliche Informationsquelle war die vom bvkj Saarland initiierte WhatsApp ${ }^{\mathrm{TM}}$-Gruppe. Sie umfasst derzeit 84 Mitglieder.

Mit der KVS besteht eine gute Vernetzung. Schwierig gestaltet sich die Zusammenarbeit mit dem öffentlichen Gesundheitswesen, v. a. mit bestimmten lokal zuständigen Gesundheitsämtern.

Anfänglich gab es keine strukturierte Kooperation mit den zuständigen Landesministerien. Im Verlauf der Pandemie änderte 
sich dies nach einer Initiative des bvkj; inzwischen werden Vertreter des bvkj und des infektiologischen Paedine Saar Netzwerkes in Beratungsprozesse eingebunden.

\section{Verbesserungsmöglichkeiten}

Der im Internet, sowohl beim Gesundheitsministerium veröffentlichte als auch beim RKI verlinkte Pandemieplan des Saarlandes ist bis einschließlich 01.11.2020 auf dem Stand vom 18.10.2006 [13]. Der Plan fokussiert ausschließlich auf Influenza und es sind keine Erfahrungen der H1N1-Pandemie eingeflossen. Er bietet den Praxen keine Planungsgrundlage, bereits nach der H1N1-Pandemie wurde auf die Notwendigkeit von fortlaufend aktualisierten Pandemieplanungen hingewiesen [3].

In der Pandemievorbereitung sehen $63 \%$ der Kollegen die dringende Notwendigkeit, die Bevorratung und Verteilung von PSA besser zu organisieren. Der Influenzapandemieplan des Saarlandes weist diese Aufgaben den Apotheken zu, die jedoch bereits zum Zeitpunkt des ersten Falls im Saarland nicht mehr lieferfähig waren. Auf der anderen Seite wurde ein Defizit in der Kommunikation gesehen, dieses betraf neben dem öffentlichen Gesundheitsdienst (ÖGD), wo einheitliche Absprachen und einheitliches Vorgehen gefordert werden, auch die Kommunikation mit Kinderbetreuungseinrichtungen, die mit der Situation (z. B. wer darf die Einrichtung besuchen, wer muss getestet werden) überfordert waren.

Neben der effektiven Versorgung mit PSA und Desinfektionsmitteln stand in der aktuellen Pandemiebewältigung wie auch bereits im Zusammenhang mit der H1N1-Pandemie das Thema der Kommunikation ganz oben auf der Agenda [1, 3]: Kommunikation und Absprachen zwischen ÖGD und kurativer Medizin, aber auch eine regelmäßige Kommunikation mit der Bevölkerung, z. B. kontinuierlicher und früher Hinweis auf Abstand, Hygiene und Alltagsmaske, Bevölkerungsinformationen zur Selbstisolation und Quarantäne, Besuch von Betreuungseinrichtungen usw.

Im Hinblick auf die Zusammenarbeit mit dem ÖGD sind Verbesserungen in Kommunikation und Informationsfluss, in der Erreichbarkeit der Gesundheitsämter für Ärzte, klare Zuständigkeiten sowie einheitliche Aussagen, sowohl innerhalb eines Amtes aber auch zwischen den Ämtern, erforderlich. Aufgrund der hausärztlichen Position werden die Praxen zu Themen wie Kontaktpersonenstatus und/oder Quarantäne kontaktiert, insbesondere wenn die Gesundheitsämter wegen Überlastung nicht erreichbar sind.

Der Verbesserungsbedarf in der Kommunikation, in enger Zusammenarbeit mit den relevanten Berufs- und Fachverbänden, war bereits in der Auswertung der H1N1-Pandemie 2010 durch den heutigen RKI-Vizepräsidenten, Herrn Professor Dr. Lars Schaade, formuliert worden [3].

Die von den saarländischen Kinder- und Jugendärzten im Rahmen der Pandemievorbereitung, Pandemiebewältigung und Zusammenarbeit mit dem ÖGD benannten Verbesserungsmöglichkeiten, sind fast vollständig bereits 2014 als Erfahrung aus der H1N1-Pandemie und der EHEC-Epidemie im Rahmen von Hausarztbefragungen identifiziert und publiziert worden [1]. In der COVID-19-Pandemie steht auch das Thema Finanzierung wieder auf der Agenda [1], aber v. a. eben die Bevorratung und Verteilung von PSA. Die Akzeptanz der Arbeitsschutz- und Hygienemaßnahmen ist in der aktuellen Pandemie deutlich höher als in der H1N1Pandemie [2].
Für die Pädiatrie typisch ist die enge Kommunikation mit Schulen und Kitas. Hier sehen die Kolleginnen und Kollegen dringenden Verbesserungsbedarf. Bei vorübergehend fehlenden konkreten Vorgaben durch die Aufsichtsbehörden sind hier eine Vielzahl von widersprüchlichen einrichtungs- oder trägereigenen Regelungen entstanden. Dies hat zum unnötigen Ausschluss der Kinder von Bildung und sozialer Teilhabe geführt sowie zu vielen medizinisch fragwürdigen „Attest-Konsultationen“.

\section{Limitationen}

Trotz der hohen Teilnahmequoten von 85 \% bei den Praxisinhabern sowie 81 \% beim nicht-ärztlichen Assistenzpersonal bleibt die Frage offen, ob die befragte Stichprobe repräsentativ war.

Auch bei Gemeinschaftspraxen wurde die Teilnahme eines Praxisinhabers erbeten, da die meisten Fragen sich auf den Bereich der Praxisorganisation bezogen. Bei den wenigen Fragen zur Person (z. B. Zugehörigkeit zur Risikogruppe gemäß RKI-Definition) kann es hierdurch zu einer Verzerrung im Antwortbild gekommen sein.

\section{FAZIT FÜR DIE PRAXIS}

- Die Kinder- und Jugendarztpraxen im Saarland haben in der ersten Welle von COVID-19 die Versorgungssicherheit aufrechterhalten.

- Das Praxismanagement wurde entsprechend der Pandemiesituation angepasst und Arbeitsschutz- und Hygieneempfehlungen wurden angenommen.

- Die Pandemie und der Lockdown in KW 12-18 haben zu Kollateralschäden bei Kindern und Jugendlichen geführt (verspätete Behandlung, Belastungssituationen \& -reaktionen, usw.).

- Moderne Medien (Messengerdienst, Videocast) können für rasche Informationsvermittlung sorgen.

- In der Pandemiebewältigung ist wie in der -vorbereitung Kommunikation zwischen allen Akteuren, v. a. dem ÖGD und der kurativen Medizin, erforderlich. Erfahrungen aus der H1N1-Pandemie wie aktuelle Pandemieplanungen, Informations- und Kommunikationsstrukturen sind offensichtlich bis heute nicht ausreichend umgesetzt und daher erneut Gegenstand von dringendem Verbesserungsbedarf.

- Die Akzeptanz von Arbeitsschutz- und Hygieneempfehlungen hat sich verbessert, zur Umsetzung bedarf es jedoch ausreichender PSA. Entsprechend sind bereits in der Pandemievorbereitung die Bevorratung und Verteilung ein zentraler Punkt, der zur Vorbereitung zukünftiger Pandemien unbedingt berücksichtigt werden muss.

- Kinderbetreuungseinrichtungen sind Dreh- und Angelpunkte im Leben von Kindern; diese müssen in Kommunikationsstrukturen eingebunden werden. Es müssen für Eltern und Einrichtungen klare und verbindliche Regelungen bestehen, sodass alle Planungssicherheit haben und auch die Kinder- und Jugendärzte Hand in Hand mitarbeiten und Eltern sowie ggf. auch Einrichtungen beraten können. 


\section{Ethische und rechtliche Anforderungen}

Alle beschriebenen Untersuchungen wurden mit Zustimmung der Ethikkommission der Ärztekammer des Saarlandes durchgeführt (Kenn.-Nr. 188/20).

Dieser Beitrag beinhaltet keine Studien an Menschen oder Tieren.

\section{Danksagung}

Der herzliche Dank gilt den saarländischen Kinder- und Jugendarztpraxen und ihren Teams, die an den beiden Befragungen teilgenommen haben. Ein besonderer Dank geht an Frau Dr. Ute Rexroth, die mir auch über Ihre Publikation hinaus Einblick in Ihre Untersuchung und gewonnen Daten gewährt hat.

\section{Interessenkonflikt}

Die Autorinnen/Autoren geben an, dass kein Interessenkonflikt besteht.

\section{Literatur}

[1] Eisele M, Hansen $\mathrm{H}$, Wagner $\mathrm{HO}$ et al. Epidemien und Pandemien in der hausärztlichen Praxis. Was können wir aus dem Schweinegrippe (H1N1)- und EHEC-Ausbruch lernen? Bundesgesundheitsblatt 2014; 57: 687-693. doi:10.1007/s00103-014-1970-z

[2] Rexroth U, Buda S. Praxismanagement und Arbeitsschutz während der Influenza-Pandemie 2009/2010 - Eine Umfrage unter 1150 Ärzten der Arbeitsgemeinschaft Influenza am Robert Koch-Institut. Gesundheitswesen 2014; 76: 670-675. doi:10.1055/s-0033-1355402

[3] Schaade L, Reuss A, Haas W et al. Pandemieplanung. Was haben wir aus der Pandemie (H1N1) 2009 gelernt? Bundesgesundheitsblatt 2010; 53: 1277-1282. doi:10.1007/s00103-010-1162-4
[4] Theiß K, Simon A, Graf N et al. Auswirkungen des Lockdowns während der ersten COVID-19 Welle auf 34 kinder- und jugendärztliche Praxen im Saarland. Monatsschr Kinderheilkd [Online ahead of print]. 2021; doi:10.1007/s00112-021-01125-9

[5] Lazzerini M, Barbi E, Apicella A et al. Delayed access or provision of care in Italy resulting from fear of COVID-19. Lancet Child Adolesc Health 2020; 4: e10-e11. doi:10.1016/s2352-4642(20)30108-5

[6] Kamrath C, Mönkemöller K, Biester T et al. Ketoacidosis in Children and Adolescents With Newly Diagnosed Type 1 Diabetes During the COVID-19 Pandemic in Germany. JAMA 2020; 324: 801-804. doi:10.1001/jama.2020.13445

[7] Snapiri O, Rosenberg Danziger C, Krause I et al. Delayed diagnosis of paediatric appendicitis during the COVID-19 pandemic. Acta Paediatr 2020; 109: 1672-1676. doi:10.1111/apa.15376

[8] Zhou X. Managing psychological distress in children and adolescents following the COVID-19 epidemic: A cooperative approach. Psychol Trauma 2020; 12: S76-S78. doi:10.1037/tra0000754

[9] Golberstein E, Wen H, Miller BF. Coronavirus Disease 2019 (COVID-19) and Mental Health for Children and Adolescents. JAMA Pediatr 2020; 174: 819-820. doi:10.1001/jamapediatrics.2020.1456

[10] Walger $P$, Heininger $U$, Knuf $M$ et al. Children and adolescents in the CoVid-19 pandemic: Schools and daycare centers are to be opened again without restrictions. The protection of teachers, educators, carers and parents and the general hygiene rules do not conflict with this. GMS Hyg Infect Control 2020; 15: Doc11. doi:10.3205/ dgkh000346

[11] Nationale Akademie der Wissenschaften Leopoldina CoronavirusPandemie: Für ein krisenresistentes Bildungssystem. 2020; Zugriff 05.08.2020

[12] Gutensohn D. "Solche Verletzungen kann sich kein Säugling selbst zufügen”. ZEIT Online 2020. Zugriff: 26.10.2020

[13] Ministerium für Justiz Gesundheit und Soziales im Saarland. Influenzapandemieplan des Saarlandes https://wwwsaarlandde/SharedDocs/ Downloads/DE/msgff/tp_gesundheitpr\%C3\%A4vention/downloads_ servicegesundheit/download_Pandemieplanpdf? blob = publicationFile\&v = 2 2006; Zugriff 01.11.2020 